\title{
Food Insecurity, Malnutrition, and the Microbiome
}

\author{
Vikram J. Christian ${ }^{1}$ (D) Keith R. Miller ${ }^{2}$ - Robert G. Martindale ${ }^{3}$ \\ Accepted: 23 October 2020 / Published online: 10 November 2020 \\ (C) Springer Science+Business Media, LLC, part of Springer Nature 2020
}

\begin{abstract}
Purpose of Review Food insecurity is defined as the disruption of food intake or eating patterns because of lack of money and other resources. Although a relationship between food insecurity and malnutrition would seem intuitive, this relationship remains unclear. This review was aimed at summarizing the emerging evidence of the relationship between food insecurity, malnutrition, and intestinal microbial changes.

Recent Findings Evidence suggests that malnutrition is certainly associated with alteration in the intestinal microbiome. Alternative theories have been proposed as to the nature of the alteration, whether that be a result of microbial immaturity, dysbiosis, or contributions from both processes.

Summary Although the evidence continues to evolve, targeted nutritional therapies in conjunction with specific microbial therapy of probiotics or prebiotics may hold great promise for the treatment of malnutrition in the clinical and community settings.
\end{abstract}

Keywords Food insecurity $\cdot$ Food desert $\cdot$ Hunger $\cdot$ Malnutrition $\cdot$ Microbiome $\cdot$ Microbial immaturity $\cdot$ Dysbiosis $\cdot$ Probiotic

\section{Introduction: Food Insecurity-Definition and Significance}

Food insecurity is defined as the disruption of food intake or eating patterns because of lack of money and other resources [1]. Accordingly, food-insecure households are defined by the United States Department of Agriculture (USDA) as households in which "access to adequate food is limited by a lack of money or other resources" [2]. In 2013, 17.5 million US households or $14.3 \%$ of all households met this definition [3]. An estimated $11.8 \%$ of US households were food-

This article is part of the Topical Collection on Gastroenterology Critical Care, and Lifestyle Medicine

Vikram J. Christian chri4793@umn.edu

1 Department of Pediatrics, Division of Gastroenterology, University of Minnesota, 2450 Riverside Ave, East Building, 6th floor, MB657, Minneapolis, MN 55454, USA

2 Department of Surgery, Division of Trauma and Surgery Critical Care, University of Louisville, Louisville, KY, USA

3 Department of Surgery, Division of Gastrointestinal and General Surgery, Oregon Health Sciences University, Portland, OR, USA insecure at least some time during the year in 2017. In 2018, $11.1 \%$ of US households were food-insecure [4].

In 2017, the USDA revised the definitions of food insecurity. The USDA defined the term "low food security" that was previously referred to as "food insecurity without hunger," as a household that reports reduced quality, variety, or desirability of diet with little or no indication of reduced food intake. The USDA defined "very low food security" that was previously referred to as "food insecurity with hunger" as a household that reports of multiple indications of disrupted eating patterns and reduced food intake [5]. The prevalence of very low food security was $4.3 \%$ in 2018 [4].

Food insecurity often correlates with residence in a food desert. The USDA defines food deserts as low-income communities that lack stores that sell healthy and affordable food. A significant portion of these communities live more than a mile from the nearest supermarket or grocery store in urban areas or 10 miles away in rural areas. In most of these cases, transportation to obtain "healthy" foods is limited. With limited options, many people living in food deserts get meals from fast-food restaurants [6].

Access to healthy and affordable food has worsened during the COVID-19 pandemic. In Georgia, the number of people now living in food-insecure areas has increased by $69 \%$ since the start of the pandemic. In Mississippi, Louisiana, and Kentucky, the 
number of residents living in food-insecure areas has increased by 36,43 , and $118 \%$, respectively, due to the rise in unemployment [7].

Food-insecure children fall sick more often and are more likely to be hospitalized. In 2009, the average pediatric hospitalization in the USA cost approximately $\$ 12,000$ [8]. Child hunger is estimated to cost the US economy in excess of $\$ 28$ billion per year. This cost is due to the fact that poorly nourished children perform less well in school and require far more long-term healthcare spending [9].

Child hunger leads to a myriad of issues for both the parent and the child (as they enter the workforce), leading to greater absenteeism, presenteeism (productivity loss), and turnover in the work force. This leads to additional employer costs as sick children often result in parent employee absences. Adult members of the workforce who experienced hunger as children are not as well prepared to perform effectively in the contemporary workforce. These workers create a workforce that is less competitive, with lower levels of educational and technical skills, and limited human capital [8].

\section{The Link between Food Insecurity and Malnutrition}

Not surprisingly, there does seem to be a link between food insecurity and malnutrition. Food insecurity is associated with low dietary quality in adults. According to one review, foodinsecure adults consume fewer fruit, vegetables, and dairy products when compared to food-secure adults. Food-insecure adults also consume less vitamins A and B6, calcium, zinc, and magnesium. Children of food-insecure households have a slightly better quality of diet (than their parent), indicating that parents effectively shield their children from compromised dietary quality [10॰].

In 2018, the United Nations Food and Agriculture Organization (FAO) released a comprehensive review of literature examining the link between food insecurity and malnutrition. Nineteen studies looking at food insecurity and childhood stunting (a well-defined indicator of childhood malnutrition) demonstrated a positive association, 3 showed mixed association, and 7 showed no association. Three studies looking at food insecurity and childhood wasting showed positive association, 1 showed mixed association, and 11 showed no association. In studies linking food insecurity and low birthweight babies, all 3 studies showed a positive association [11]. Overall, they concluded that the evidence was mixed and that "high quality longitudinal data is required to better understand the true nature of the relationship."

\section{Food Deprivation and the Microbiome}

In 2015, Genton et al. reviewed the available literature to summarize the impact of depleted nutritional states on the gut microbiota (as well as structure and function of the gut epithelium, the gut-associated lymphoid tissue and the enteric nervous system). Genton concluded that gut microbiota composition and function was indeed altered in nutritionally depleted states [12]. This conclusion was supported by the study by Monira et al. that demonstrated a higher fecal proportion of Proteobacteria and a lower fecal proportion of Bacteroidetes in malnourished children when compared with healthy children [13]. Genton also cited Armougom et al. who demonstrated higher fecal proportion of Methanobrevibacter smithii in anorectic patients. The authors note that $M$. smithii is known to convert carbon dioxide and hydrogen to methane and hence could optimize energy harvesting in the midst of starvation [14]. Therefore, there is a change that occurs in the microbiome in a food deprived or starvation state.

\section{Malnutrition and Microbial Immaturity}

Malnutrition is associated with "immaturity of the microbiome." This concept of microbial maturity stems from a 2012 study that characterized the bacterial species present in the fecal samples of 531 individuals. DNA was extracted from each fecal sample and bacterial 16S ribosomal RNA (16S-rRNA) genes were amplified to identify the various phylogenetic types that were present in each sample. By studying 16S-rRNA datasets, the authors found a variation in the taxonomic/phylogenetic composition of fecal communities across the ages. The authors noted that the phylogenetic composition in infants gradually transitioned toward a composition that resembled that of adults within the first 3 years of life. Through further analysis, the authors determined that representation of specific bacterial taxa was noted to vary significantly across the ages. Bifidobacterium was one among other bacterial taxa found to be disproportionately dominant in infant fecal samples. The authors list a total of 126 bacterial species, the representation of which varied significantly with age. Increased bacterial diversity with age was noted. By means of genetic sequencing and metagenomic analysis, the authors found ageassociated changes in representation of genes involved in nutrient biosynthesis and metabolism. This change in phylogenetic composition and genetic representation with age are considered to be signs of microbial maturity [15].

In 2013, Smith et al. published data from Malawi where they followed 317 Malawian twin pairs for 36 months. The authors focused on twin pairs where the growth was discordant, where one member of the pair had normal growth and nutrition and the other member of the twin pair had kwashiorkor. Both twins were fed a ready to use therapeutic food (RUTF) which comprised of peanut paste, sugar, vegetable oil, and milk 
fortified with vitamins and minerals. RUTF is recommended by the World Health Organization (WHO) for the treatment of children with uncomplicated severe acute malnutrition [16]. The authors found that there was transient maturation of metabolic function in the kwashiorkor microbiomes, but this maturation regressed when the therapeutic food was discontinued. The authors then transplanted the previously frozen fecal communities of these discordant twins into gnotobiotic mice. The mice transplanted with the microbiome from the kwashiorkor twin experienced more weight loss when fed a Malawian diet. The pairs of mice were then fed the RUTF for the 2 weeks. With this diet, both groups of mice increased weight. By means of gas chromatography-mass spectrometry, the authors demonstrated increase in short-chain fatty acids and other products of metabolism in cecal and fecal samples when the mice were fed the RUTF [17•].

A study published by Subramaniam et al. demonstrated persistent gut microbiota immaturity in malnourished Bangladeshi children. To do this, a machine-learning-based approach was applied to $16 \mathrm{~S}$ ribosomal RNA data sets generated from monthly fecal samples obtained from children who exhibited healthy growth. This data was incorporated into a model that computed a relative microbiota maturity index and a microbiota for age $\mathrm{Z}$ score. Relative microbiota immaturity of a child was defined as the difference in the microbiota age of the child with that of healthy children of similar chronologic age. The microbiota for age Z-score was derived from this value. Analyzing children with severe acute malnutrition (SAM) and moderate acute malnutrition (MAM), the authors found that SAM was associated with significant relative microbiota immaturity [18].

Children with severe acute malnutrition were further enrolled in a randomized trial of two food interventions (RUTF vs Khichuri Halwa, a local food option). The microbial maturity of children in each arm of the trial were compared before the trial, at the end of the intervention, less than 3 months after intervention and greater than 3 months after intervention. The authors found that although growth improved, the microbiota immaturity was only partially improved with both nutritional interventions. The authors concluded that either prolonged interventions with therapeutic foods or the addition or supplementation of gut microbes may be needed to achieve enduring "repair" of the microbial immaturity [18]. Taken together, the above studies suggest that microbial immaturity can result in alterations in the way food sources are metabolized. Provision of nutrient alone can encourage maturation over prolonged administration but, potentially, by altering the microbiome itself this process could be expedited.

\section{Malnutrition and Dysbiosis}

An alternative theory relevant to the malnourished microbiome has been suggested by Million et al. involving a qualitative shift (irreversible loss of gut microbes) rather than microbiota immaturity as the key contributor in characterizing children with SAM [19]. This altered microbial composition is referred to as dysbiosis [20]. Dysbiosis and the resulting microbial metabolites (metabolome) may play a role in the development of obesity, insulin resistance, non-alcoholic fatty liver disease, type 2 diabetes mellitus, and atherosclerosis among other conditions [21]. Dysbiosis has been linked to epigenetic changes (mutations in NOD2, IL23R, ATG16L, and IGRM), lifestyle, early microbial colonization, and medical practices (such as the use of antibiotics) [22]. More recently, a link between dysbiosis and malnutrition has become more evident [20]. Hashimoto et al. demonstrated that tryptophan deficiency from a protein-free diet can lead to changes in the colonic microbiota. The authors also demonstrated that this may result in a more severe colitis in mice following exposure to dextran sodium sulfate, an intestinal irritant that causes colitis [23]. Therefore, malnutrition may bring about qualitative changes in the microbiome, and this may lead or contribute toward a disease state.

Similarly, recently published data in the New England Journal of Medicine reported that enteric dysfunction played a significant role in childhood undernutrition. The authors elucidated and reported a possible causal relationship between growth stunting and enteric bacteria [24]. In this study, 110 children living in a low-income neighborhood in Bangladesh who had not benefited from nutritional intervention underwent upper endoscopy and small bowel biopsy confirming the diagnosis of Environmental Enteric Dysfunction (EED). A large series of plasma and duodenal protein analyses were performed and found to be associated with small bowel and fecal bacterial strains. The bacterial strains associated with refractory undernutrition were then placed in gnotobiotic mice and resulted in enteropathy. These results support the causal relationship between specific small intestinal bacteria and enteropathy, rather than microbial immaturity.

\section{Dysbiosis and the Gut-Brain Axis}

In addition to enteropathies, dysbiosis potentially contributes in part to other maladies. Poor access to "healthy" food in early life may be a stepping-stone to several neurologic conditions. Numerous factors shape the microbiome in developing children including genetics, stress, diet, acute and chronic infections, medications, and environmental factors [25•]. Autism, anxiety, anti-social behavior, and depression have recently been associated with an altered microbiome. Stress influences the gut-brain axis via several well-described mechanisms [26]. Much of this early work comes from studying gnotobiotic mice, various foods and stress and how these microbiome-induced changes can result in several cognitive and emotional changes attributed at least in part secondary to inflammatory responses [25, 27, 28]. 


\section{Microbiome-Targeted Therapies}

With the objective to "repair" microbial immaturity, Gehrig et al. colonized gnotobiotic mice with bacteria from children with SAM who were transitioning to MAM. Foods were offered to assess what might be able to promote microbial maturity. The group formulated microbiome directed complementary foods (MDCF's) that were made using culturally acceptable, affordable, and readily available ingredients. The authors selected ingredients that were more likely to promote microbial maturity [29••]. The authors then proceeded with a randomized double-blinded clinical trial in Dhaka, Bangladesh, in which 63 children were enrolled. The authors compared a rice and lentil-based ready-to-use supplementary food (RUSF) to three MDCF prototypes. MDCF-1 and MDCF-2 contained chickpea flour, soy flour, peanut flour, and banana in different concentrations. MDCF-3 contained chickpea flour and soy flour. Each formulation was equivalent in calories and protein content. The children were fed 2 servings a day of each trial food on top of their regular diet. The authors were able to conclude that MDCF-2 led to increased levels of biomarkers and mediators of growth, bone formation, neurodevelopment, and immune function toward a state resembling healthy children [29••]. This approach would suggest that specific foods can promote the maturation of the microbiome, thereby potentially improving overall health.

\section{Probiotics}

A recent systematic review and meta-analysis examined the role of probiotics in preterm, low birth weight infants. This review found that combinations of Lactobacillus spp. and Bifidobacterium spp. reduced all-cause mortality when compared with placebo. Among the studies with high or moderate certainty evidence relative to placebo, combinations of Lactobacillus spp. and Bifidobacterium spp., Bifidobacterium animalis subsp. lactis, L. reuteri, and Lactobacillus rhamnosus significantly reduced severe necrotizing enterocolitis. This study also found that combinations of Lactobacillus spp. and Bifidobacterium spp. and S. boulardii reduced the mean number of days to reach full feeds [30•]. This suggests that specific strains of probiotics can potentially address the qualitative shift that occurs in the malnourished microbiome.

\section{Conclusion}

Food insecurity, or the disruption of food intake or eating patterns because of lack of financial resources and other related resources such as proximity to reliable fresh foods, lack of transportation, or adequate refrigeration, is nationally prevalent and may be increasing in prevalence in the setting of the current COVID-19 pandemic. There does appear to be a link between food insecurity and malnutrition, although certain indices of malnutrition do not correlate, as expected, with food insecurity. Certainly, food insecurity and alterations in the developing microbiome are inextricably associated. Malnutrition may be linked to microbiota immaturity and/or dysbiosis. Certain strains of bacteria from the poorly nourished children may be contributory to the inflammatory cascade which limits the benefit of the supplemental nutrition and consequently limits the child's growth and development. Targeted nutritional therapies in conjunction with specific microbial therapy of probiotics or prebiotics may hold promise for treatment of malnutrition in the clinical and community settings. Although the evidence for these approaches remains in their infancy, the potential for global impact is substantial and encouraging.

\section{Compliance with Ethical Standards}

Conflict of Interest Vikram J. Christian declares that he has no conflict of interest.

Keith R. Miller receives honorarium for Nestle educational fellowship.

Robert G. Martindale declares that he has no conflict of interest.

\section{References}

Papers of particular interest, published recently, have been highlighted as:

- Of importance

•- Of major importance

1. Nord M, Andrews M, Carlson S. Household food security in the United States, 2005. 2006. https://www.ers.usda.gov/webdocs/ publications $/ 45655 / 29206$ err29_002.pdf?v=1662.1. Accessed 5 Nov 2020.

2. Coleman-Jensen A, McFall W, Nord M. Food insecurity in households with children: prevalence, severity, and household characteristics, 2010-11. 2013. https://www.ers.usda.gov/publications/ pubdetails/?pubid=43765. Accessed 5 Nov 2020.

3. Coleman-Jensen A, Gregory C, Singh A. Household food security in the United States in 2013. 2014. https://www.ers.usda.gov/ webdocs/publications/45265/48787_err173.pdf. Accessed 5 Nov 2020.

4. Coleman-Jensen A, Rabbitt M, Gregory C, Singh A. Household food security in the United States in 2018. 2019. https://www.ers. usda.gov/publications/pub-details $/$ ?pubid $=94848$. Accessed 5 Nov 2020.

5. Definitions of Food Insecurity. USDA Economic Research Service. https://www.ers.usda.gov/topics/food-nutritionassistance/foodsecurity-in-the-us/definitions-of-food-security.aspx. Accessed 5 Nov 2020.

6. Ver Ploeg M, Breneman V, Farrigan T, Hamrick K, Hopkins D, Kaufman P, et al. Access to affordable and nutritious food-measuring and understanding food deserts and their consequences: report to Congress. https://www.ers.usda.gov/publications/pub-details/? pubid $=42729$. Accessed 5 Nov 2020. 
7. Meyersohn N. Groceries were hard to find for millions. Now it's getting even worse. CNN Business; 2020. https://www.cnn.com/ 2020/06/09/business/food-desertscoronavirus-grocery-stores/ index.html. Accessed 5 Nov 2020.

8. Cook J, Jeng K. Child Food Insecurity: The Economic Impact on our Nation. 2009. https://www.nokidhungry.org/sites/default/files/ child-economy-study.pdf. Accessed 5 Nov 2020.

9. Brown L, Shepard D, Martin T, Orwat J. The Economic Cost of Domestic Hunger: Estimated Annual Burden to the United States. 2007. http:/us.stop-hunger.org/files/live/sites/stophungerus/files/ HungerPdf $/$ Cost $\% 20$ of $\% 20$ Domestic $\% 20$ Hunger $\% 20$ Report $\% 20$ tcm150-155150.pdf. Accessed 5 Nov 2020.

10. Hanson KL, Connor LM. Food insecurity and dietary quality in US adults and children: a systematic review. Am J Clin Nutr. 2014;100(2):684-92. https://doi.org/10.3945/ajcn.114.084525. Comprehensive systematic review on dietary quality in food secure households.

11. Maitra C. A review of studies examining the link between food insecurity and malnutrition. 2018. http://www.fao.org/3/ CA1447EN/ca1447en.pdf. Accessed 5 Nov 2020.

12. Genton L, Cani PD, Schrenzel J. Alterations of gut barrier and gut microbiota in food restriction, food deprivation and protein-energy wasting. Clin Nutr. 2015;34(3):341-9. https://doi.org/10.1016/j. clnu.2014.10.003.

13. Monira S, Nakamura S, Gotoh K, Izutsu K, Watanabe H, Alam NH, et al. Gut microbiota of healthy and malnourished children in Bangladesh. Front Microbiol. 2011;2:228. https://doi.org/10.3389/ fmicb.2011.00228.

14. Armougom F, Henry M, Vialettes B, Raccah D, Raoult D. Monitoring bacterial community of human gut microbiota reveals an increase in Lactobacillus in obese patients and methanogens in anorexic patients. PLoS One. 2009;4(9):e7125. https://doi.org/10. 1371/journal.pone.0007125.

15. Yatsunenko T, Rey FE, Manary MJ, Trehan I, Dominguez-Bello MG, Contreras M, et al. Human gut microbiome viewed across age and geography. Nature. 2012;486(7402):222-7. https://doi.org/10. 1038/nature11053.

16. Essential Nutrition Actions: mainstreaming nutrition through the life-course. Geneva: World Health Organization; 2019.

17. Smith MI, Yatsunenko T, Manary MJ, Trehan I, Mkakosya R, Cheng J, et al. Gut microbiomes of Malawian twin pairs discordant for kwashiorkor. Science. 2013;339(6119):548-54. https://doi.org/ $10.1126 /$ science. 1229000 . The authors establish the role of the microbiome in malnutrition among Malawian twin pairs.

18. Subramanian S, Huq S, Yatsunenko T, Haque R, Mahfuz M, Alam $\mathrm{MA}$, et al. Persistent gut microbiota immaturity in malnourished Bangladeshi children. Nature. 2014;510(7505):417-21. https:// doi.org/10.1038/nature13421.

19. Million M, Raoult D. Gut dysbiosis in severe acute malnutrition is not an immaturity: The irreversible quantitative-qualitative paradigm shift. Human Microbiomed J. 2020;15:100067. https://doi. org/10.1016/j.humic.2019.100067.
20. Kane AV, Dinh DM, Ward HD. Childhood malnutrition and the intestinal microbiome. Pediatr Res. 2015;77(1-2):256-62. https:// doi.org/10.1038/pr.2014.179.

21. Schroeder BO, Bäckhed F. Signals from the gut microbiota to distant organs in physiology and disease. Nat Med. 22(10):1079-89. https://doi.org/10.1038/nm.4185.

22. Round JL, Mazmanian SK. The gut microbiota shapes intestinal immune responses during health and disease. Nat Rev Immunol. 2009;9(5):313-23. https://doi.org/10.1038/nri2515.

23. Hashimoto T, Perlot T, Rehman A, Trichereau J, Ishiguro H, Paolino M, et al. ACE2 links amino acid malnutrition to microbial ecology and intestinal inflammation. Nature. 2012;487(7408):47781. https://doi.org/10.1038/nature11228.

24. Chen RY, Kung VL, Das S, et al. Duodenal microbiota in stunted undernourished children with enteropathy. N Engl J Med. 383(4): 321-33. https://doi.org/10.1056/NEJMoa1916004.

25. Cowan CSM, Dinan TG, Cryan JF. Annual research review: critical windows - the microbiota-gut-brain axis in neurocognitive development. J Child Psychol Psychiatry. 2020;61(3):353-71. https:// doi.org/10.1111/jcpp.13156. The authors make a case for the microbiota-gut-brain hypothesis.

26. Callaghan BL, Cowan CS, Richardson R. Treating generational stress: effect of paternal stress on development of memory and extinction in offspring is reversed by probiotic treatment. Psychol Sci. 2016;27(9):1171-80. https://doi.org/10.1177/ 0956797616653103 .

27. Bermúdez-Humarán LG, Salinas E, Ortiz GG, Ramirez-Jirano LJ, Morales JA, Bitzer-Quintero OK. From probiotics to psychobiotics: live beneficial bacteria which act on the brain-gut axis. Nutrients. 2019;11(4). https://doi.org/10.3390/nu11040890.

28. Bienenstock J, Kunze W, Forsythe P. Microbiota and the gut-brain axis. Nutr Rev. 2015;73(Suppl 1):28-31. https://doi.org/10.1093/ nutrit/nuv019.

29.• Gehrig JL, Venkatesh S, Chang HW, et al. Effects of microbiotadirected foods in gnotobiotic animals and undernourished children. Science. 2019;365(6449). https://doi.org/10.1126/science.aau4732 The authors formulated microbiome directed complementary foods and showed that these foods repaired the microbial immaturity that is present in children with severe acute malnutrition.

30. Morgan RL, Preidis GA, Kashyap PC, Weizman AV, Sadeghirad B. McMaster probiotic Pe, and Synbiotic work group. Probiotics reduce mortality and morbidity in preterm, low-birth-weight infants: a systematic review and network meta-analysis of randomized trials. Gastroenterology. 2020. https://doi.org/10.1053/j.gastro. 2020.05.096 Thorough systematic review on the data behind clinical application of probiotics.

Publisher's Note Springer Nature remains neutral with regard to jurisdictional claims in published maps and institutional affiliations. 